\title{
Angular correlations and coherent parton branching
}

\author{
F. Hautmann* and H. Jung ${ }^{\dagger}$ \\ ${ }^{*}$ Oxford University, Theoretical Physics Department, Oxford OX1 3NP \\ ${ }^{\dagger}$ Deutsches Elektronen Synchrotron, D-22603 Hamburg
}

\begin{abstract}
We discuss coherent gluon radiation from space-like branching and its role in the Monte Carlo simulation of initial-state parton showers at high-energy colliders. We study effects of coherence due to non-collinear emission, and their potential relevance for the associated jet distributions.
\end{abstract}

Keywords: Collider physics, jets, QCD.

PACS: $12.38 .-$ t; 13.87. -a.

Studies of final states containing multiple hadron jets at the forthcoming LHC require realistic event simulation by parton shower Monte Carlo [1], including validation and tuning of the event generators using multi-jet data from Tevatron and HERA, see e.g. [2, $3,4]$.

Standard parton-shower generators, such as HERWIG and PYTHIA, are based on collinear evolution of the jets developing (both "forwards" and "backwards") from the hard event. Besides small-angle, incoherent gluon emission, the approach of these generators also incorporates coherent soft-gluon emission from partonic lines carrying longitudinal momentum fraction $x \sim \mathscr{O}(1)$. The phenomenological relevance of this treatment has been emphasized by extensive collider data studies [5].

However at the LHC, due to the phase space opening up for large center-of-mass energies, jet production enters a new regime with a great many events characterized by multiple hard scales, in which (a) effects of emissions that are not collinearly ordered become increasingly non-negligible, and (b) coherence effects set in from space-like partons carrying momentum fractions $x \ll 1$ (Fig. 1) [6]. These effects are not included in standard shower Monte Carlo generators. It is not at all obvious that the approximations involved in Monte Carlo that have successfully served for event simulation in past collider experiments will be up to the new situation.

The theoretical framework to take account of non-collinear emission and coherence in the space-like branching requires the introduction of partonic distributions unintegrated not only in the longitudinal momenta but also in the transverse momenta $[7,8,9,10,11]$. The corrections to collinear ordering correspond to higher-order radiative terms in the associated jet distributions that are logarithmically enhanced in the ratio $\sqrt{s} / E_{T}$ of the total energy $\sqrt{s}$ to the jet transverse energy. An alternative approach to non-collinear gluon radiation is based on showers of color dipoles [12] and is also being applied to the initial-state jet $[13,14]$. See [15] for a study of critical issues in the relation of this approach with the parton formulation. Either at parton or dipole level, open questions involve methods for properly combining contributions from infrared regions with highenergy subgraphs, for which we expect techniques such as those in [16] to be relevant. 
An overview of issues on unintegrated distributions and references may be found in [11].

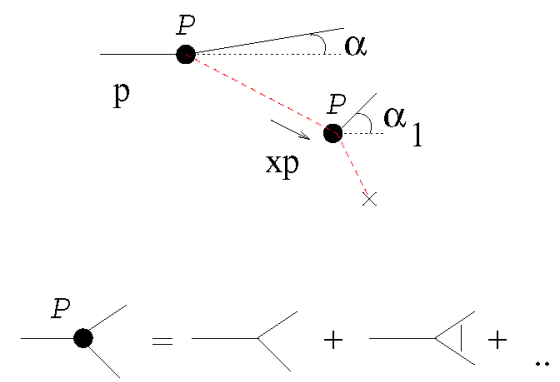

FIGURE 1. (top) Coherent radiation in the space-like parton shower for $x \ll 1$; (bottom) the unintegrated splitting function $\mathscr{P}$, including small- $x$ virtual corrections.

The effects of coherent space-like branching are investigated in [6] for angular and momentum correlations in multi-jet final states. For a multi-jet event, consider for instance the distribution in the azimuthal angle $\Delta \phi$ between the two hardest jets. At the LHC such measurements may become accessible relatively early and be used to probe the description of complex hadronic final states by QCD and Monte Carlo generators. Experimental data on $\Delta \phi$ correlations are available from the Tevatron [3] and from Hera [4]. While the Tevatron measurements are dominated [6] by leading-order QCD processes and are reasonably well described both by collinear showers (HERWIG and the new tuning of PYTHIA $[2,3]$ ) and by fixed-order NLO calculations, the Hera $\Delta \phi$ measurements probe higher orders in the dynamics of color emission, and present a more complex case, likely to be closer to the situation at the LHC.

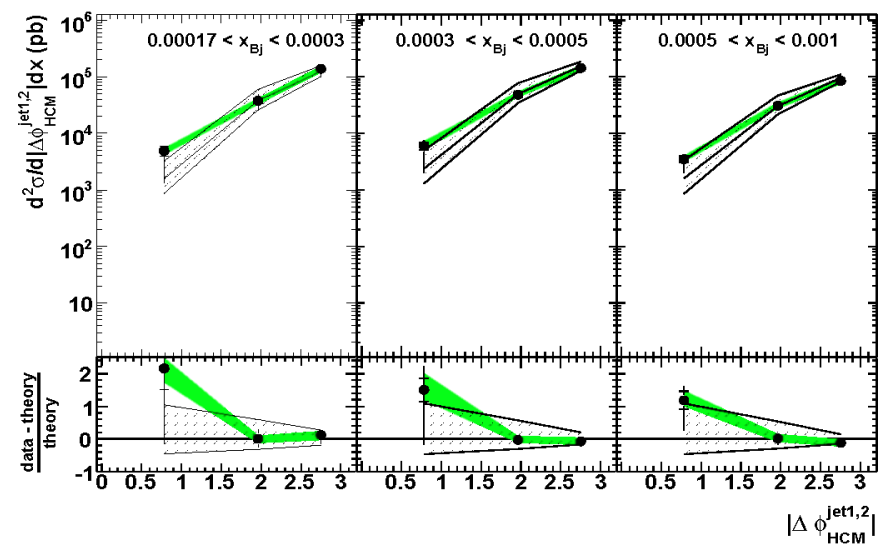

FIGURE 2. Measurement of 3-jet cross section [4] versus azimuthal separation between the two highest- $E_{T}$ jets, compared with NLO results.

In particular, it is noted in $[6,17]$ that di-jet $\Delta \phi$ correlations are affected by sizeable sub-leading corrections, resulting in large theoretical uncertainties at NLO. Analogous effects are observed [4] in the three-jet cross section (Fig. 2 [4]) particularly for the 
small- $\Delta \phi$ and small- $x$ bins. The large corrections arise from regions with three wellseparated hard jets in which the parton lines in the initial state decay chain are not ordered in transverse momentum. These corrections can be treated and summed to all orders, including coherence effects, by parton branching [6], using matrix elements (ME) and distributions (u-pdf) at fixed transverse momentum $\mathrm{k}_{\perp}$ according to the factorization [8]. Fig. 3 compares $\mathrm{k}_{\perp}$-shower (CASCADE [18]) and collinear-shower (HERWIG) results with the measurements [4]. The shape of the angular distribution is described well by the $\mathrm{k}_{\perp}$-shower. On the other hand, while the HERWIG K-factor in Fig. 3 allows one to get the normalization in the two-jet region approximately correct, the shape is not well described by HERWIG as $\Delta \phi$ decreases.
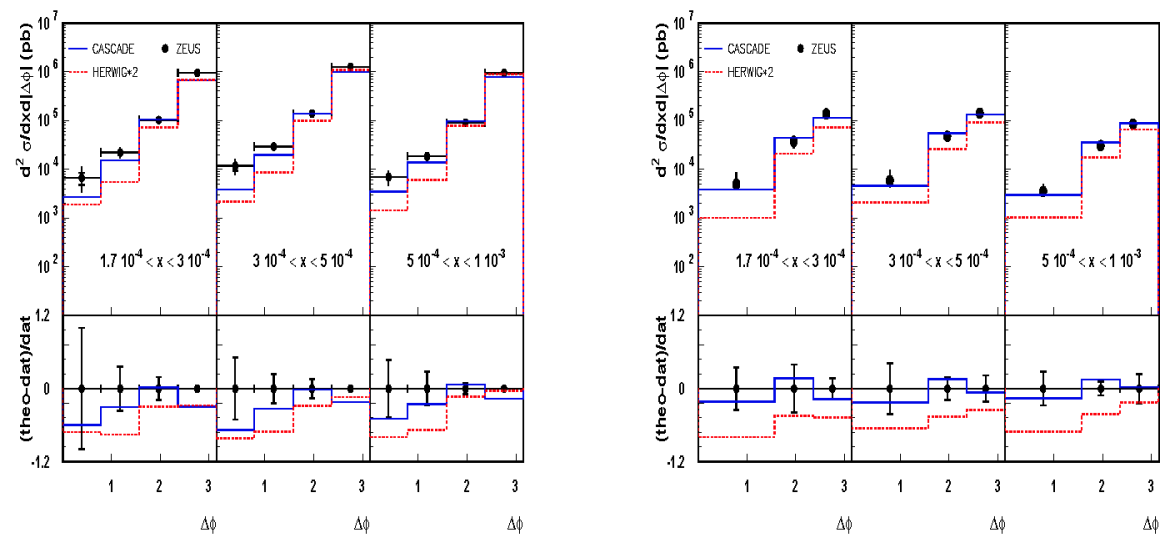

FIGURE 3. Angular jet correlations [6] from $k_{\perp}$-shower (CASCADE) and collinear-shower (HERWIG), compared with data [4]: (left) di-jet cross section; (right) three-jet cross section. The HERWIG results are multiplied by a K-factor equal to 2 to give the correct normalization in the two-jet region.

The correlations in the jets' azimuthal distances and in their transverse-momentum imbalance are sensitive to the large- $\mathrm{k}_{\perp}$ tail in the hard matrix element [19]. Fig. 4 illustrates the relative contribution of $\mathrm{ME}$ and u-pdf to the result, showing different approximations to the azimuthal dijet distribution normalized to the back-to-back cross section. The solid curve is the full result. The dashed curve is obtained from the same unintegrated pdf's but by taking the collinear approximation in the hard matrix element. The dashed curve drops much faster than the full result as $\Delta \phi$ decreases, indicating that the high- $k_{\perp}$ component in the hard $\mathrm{ME}$ is necessary to describe jet correlations for small $\Delta \phi$. The dotted curve is the result obtained from the unintegrated pdf without any resolved branching. This represents the contribution of the intrinsic $k_{\perp}$ distribution only, corresponding to nonperturbative, predominantly low- $k_{\perp}$ modes. That is, in the dotted curve one retains an intrinsic $k_{\perp} \neq 0$ but no effects of coherence. We see that the resulting jet correlations in this case are down by an order of magnitude. The inclusion of the perturbatively computed high- $\mathrm{k}_{\perp}$ correction distinguishes the calculation [6] of multijet cross sections from other shower approaches (see e.g. [20]) that include transverse 
momentum dependence in the pdfs but not in the matrix elements.

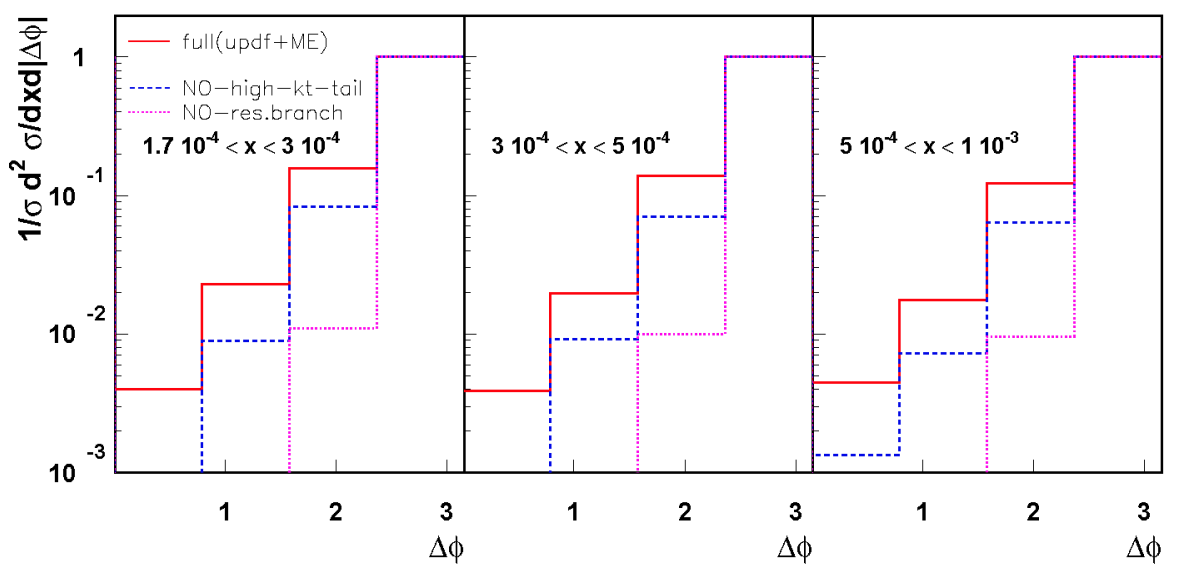

FIGURE 4. The dijet azimuthal distribution [6] normalized to the back-to-back cross section: (solid) full result (u-pdf $\oplus \mathrm{ME}$ ); (dashed) no finite- $k_{\perp}$ correction in $\mathrm{ME} \mathrm{(u-pdf} \oplus \mathrm{ME}_{\text {collin. }}$ ); (dotted) u-pdf with no resolved branching.

The coherence effects just discussed show up in the region of small $\Delta \phi$. The region of large $\Delta \phi$, on the other hand, is dominated by soft and collinear radiation [21]. But note that this region is also possibly affected by further, somewhat unexpected effects, arising from endpoint singularities [22], and possibly Coulomb exchange [23].

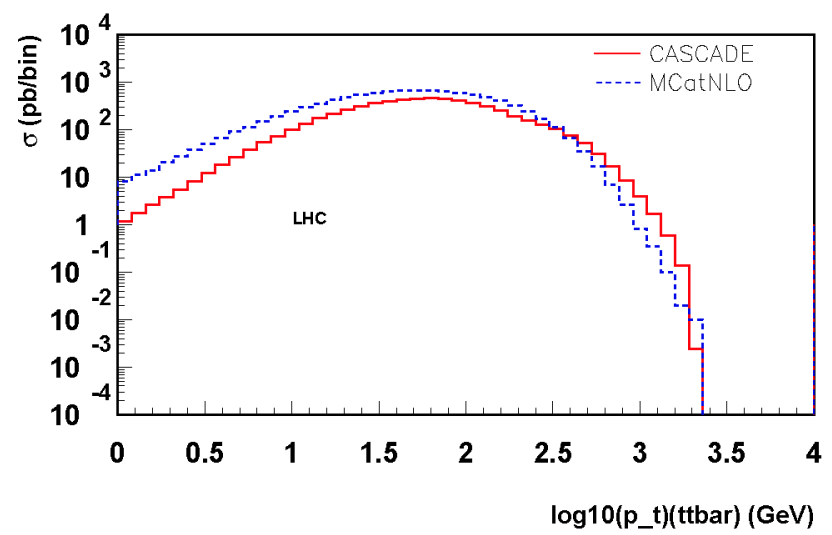

FIGURE 5. Transverse momentum distribution of top quark pairs [6] at the LHC from $\mathrm{k}_{\perp}$-shower (CASCADE) and NLO+shower (MC@NLO).

Besides jet final states, the corrections to collinear-ordered showers described in this article are also relevant to heavy particle production $[8,24,25,26]$. In Fig. 5 is the result of a numerical calculation for the transverse momentum spectrum of top-antitop pair production at the LHC. Small- $x$ effects are not large in this case. Rather, this process 
illustrates how the showering works all the way up to virtualities on the order of the top quark mass, and finite $x$. Note that even at LHC energies the transverse momentum distribution of top quark pairs calculated from the $\mathrm{k}_{\perp}$-shower is similar to what is obtained from a full NLO calculation (including parton showers, MC@NLO), with the $\mathrm{k}_{\perp}$-shower giving a somewhat harder spectrum.

\section{ACKNOWLEDGMENTS}

We thank the organizers of the conference for the invitation and the nice atmosphere at the meeting.

\section{REFERENCES}

1. See for instance S. Höche, F. Krauss, N. Lavesson, L. Lönnblad, M. Mangano, A. Schälicke and S. Schumann, hep-ph/0602031, HERA and the LHC Workshop Proceedings.

2. M.G. Albrow et al. [TeV4LHC QCD Working Group], [arXiv:hep-ph/0610012].

3. V.M. Abazov et al. [D0 Collaboration], Phys. Rev. Lett. 94 (2005) 221801 [arXiv:hep-ex/0409040].

4. S. Chekanov et al. [ZEUS Collaboration], Nucl. Phys. B 786 (2007) 152 [arXiv:0705.1931 [hep-ex]].

5. B.R. Webber, lectures at the Parma Summer School SNFT07, September 2007.

6. F. Hautmann and H. Jung, JHEP 0810 (2008) 113 [arXiv:0805.1049 [hep-ph]].

7. M. Ciafaloni, Nucl. Phys. B296 (1988) 49.

8. S. Catani, M. Ciafaloni and F. Hautmann, Phys. Lett. B242 (1990) 97; Nucl. Phys. B366 (1991) 135; Phys. Lett. B307 (1993) 147.

9. G. Marchesini and B.R. Webber, Nucl. Phys. B386 (1992) 215.

10. B. Andersson, G. Gustafson and J. Samuelsson, Nucl. Phys. B467 (1996) 443.

11. F. Hautmann and H. Jung, arXiv:0808.0873 [hep-ph]; arXiv:0712.0568 [hep-ph].

12. G. Gustafson, Phys. Lett. B175 (1986) 453.

13. L. Lönnblad and M. Sjödahl, JHEP 0505 (2005) 038; JHEP 0402 (2004) 042; G. Gustafson, L. Lönnblad and G. Miu, JHEP 0209 (2002) 005.

14. C. Flensburg, G. Gustafson and L. Lönnblad, arXiv:0807.0325 [hep-ph].

15. Yu.L. Dokshitzer and G. Marchesini, arXiv:0809.1749 [hep-ph].

16. J.C. Collins and F. Hautmann, Phys. Lett. B 472 (2000) 129; JHEP 0103 (2001) 016; F. Hautmann, Nucl. Phys. B604 (2001) 391; hep-ph/9708496.

17. F. Hautmann and H. Jung, arXiv:0804.1746 [hep-ph], in Proceedings of the 8th International Symposium on Radiative Corrections RADCOR2007 (Florence, October 2007).

18. H. Jung, Comput. Phys. Commun. 143 (2002) 100 [arXiv:hep-ph/0109102].

19. S. Catani and F. Hautmann, Nucl. Phys. B427 (1994) 475; Phys. Lett. B315 (1993) 157.

20. S. Höche, F. Krauss and T. Teubner, arXiv:0705.4577 [hep-ph].

21. A. Banfi, M. Dasgupta and Y. Delenda, Phys. Lett. B 665 (2008) 86 [arXiv:0804.3786].

22. F. Hautmann, Phys. Lett. B 655 (2007) 26; arXiv:0708.1319 [hep-ph].

23. J.R. Forshaw, A. Kyrieleis and M.H. Seymour, arXiv:0808.1269 [hep-ph].

24. H. Jung, Mod. Phys. Lett. A 19 (2004) 1; F. Hautmann, Phys. Lett. B 535 (2002) 159.

25. M. Deak et al., contribution at HERA-LHC Workshop, CERN, May 2008.

26. F.I. Olness, contribution at HERA-LHC Workshop, CERN, May 2008; S. Berge, P.M. Nadolsky, F.I. Olness and C.P. Yuan, hep-ph/0508215; M. Dasgupta and Y. Delenda, JHEP 0608 (2006) 080. 
Copyright of AIP Conference Proceedings is the property of American Institute of Physics and its content may not be copied or emailed to multiple sites or posted to a listserv without the copyright holder's express written permission. However, users may print, download, or email articles for individual use. 